\title{
PLANEJAMENTO dA ASSIStência de ENFERMAGEM A PACIENTES PORTADORES DE QUEIMADURA UTILIZANDO UM SOFTWARE: APLICAÇÃO EM QUATRO PACIENTES
}

Maria Celia Barcellos Dalri ${ }^{1}$

Emília Campos de Carvalho

Dalri MCB, Carvalho EC. Planejamento da assistência de enfermagem a pacientes portadores de queimadura utilizando um software: aplicação em quatro pacientes. Rev Latino-am Enfermagem 2002 novembro-dezembro; 10(6):787-93.

Com o objetivo de avaliar a aplicabilidade de um software (PROGQUEM) quanto à execução do processo de enfermagem a pacientes portadores de queimaduras, utilizamos o método de estudo de caso. Foram avaliados quatro sujeitos internados em uma Unidade de Queimados. Os casos foram inseridos no programa de computador onde o planejamento da assistência foi realizado. O software mostrou-se adequado, propiciando o planejamento da assistência em tempo reduzido.

DESCRITORES: processos de enfermagem, computadores, enfermagem, queimaduras

\section{PLANNING THE NURSING CARE TO BURN PATIENTS BY THE USE OF A SOFTWARE: APPLICATION IN SAMPLE OF FOUR PATIENTS}

In order to evaluate the applicability of a Software to the execution of a nursing process of care to burn patients, a case study method has been used. Four (4) subjects who had been admitted to a burn unit were evaluated. All the four cases were inserted into a computer program where the plan of nursing care was executed. The Software was adequate, enabling the planning of the nursing care in a reduced time.

DESCRIPTORS: nursing process, informatics and nursing, computers and nursing, burns

\section{PLANEACIÓN de LA ATENGIÓN de ENFERMERÍA A PACIENTES CON QUEMADURAS UTILIZANDO UN SOFTWARE; APLICACIÓN EN CUATRO PACIENTES}

Con el objetivo de evaluar la aplicabilidad de un software (PROGQUEM) a la hora de ejecutar el proceso de atención de enfermería a pacientes con quemadura, utilizamos el método de estudio de caso. Analizamos cuatro sujetos internados en la unidad de quemados. Incluimos estos casos en el programa de ordenador en el cual se realizó la planeación de la atención. El software demostró que es adecuado facilitando la planeación de la atención dentro de un tiempo reducido.

DESCRIPTORES: proceso de enfermería, computadores, enfermería, quemaduras

\footnotetext{
${ }^{1}$ Professor Doutor, e-mail: macdalri@eerp.usp.br; ${ }^{2}$ Professor Titular. Escola de Enfermagem de Ribeirão Preto da Universidade de São Paulo,
} Centro Colaborador da OMS para o desenvolvimento da pesquisa em enfermagem 


\section{INTRODUÇÃO}

$\boldsymbol{A}$ revolução da informação, com o advento dos computadores presentes na nossa vida diária, vem provocando mudanças nos hábitos e valores de vida. A aplicação da informática na área da saúde é fato concreto e encontra-se em desenvolvimento acelerado, tanto em nível nacional como internacional, contribuindo para mudanças nas estruturas organizacionais dos hospitais, estimulando profundas transformações ${ }^{(1)}$.

Diante desse contexto, o enfermeiro se depara com desafio decisivo: instrumentalizar-se para integrar novas tecnologias na enfermagem pela sistematização de suas ações e fundamentação técnico-científica do conhecimento.

À medida que ocorre o avanço do conhecimento técnico-científico na área da saúde, a enfermagem não poderia ficar para trás. Encontrar caminhos que possam responder e sustentar uma prática de enfermagem, fundamentada em conhecimento científico, é da responsabilidade de cada um de nós enfermeiros.

As atividades de competência da enfermagem têm ficado cada vez mais definidas pelos órgãos oficiais de legislação da profissão. Hoje percebemos a ênfase que se tem dado, por parte das enfermeiras, à importância na documentação e registro do plano de cuidados de saúde ${ }^{(2)}$.

Estudos realizados nas décadas de 60, 70 e 80 nos Estados Unidos relatam o impacto dos computadores no cuidado de enfermagem, dizendo que as enfermeiras americanas começaram a identificar o potencial dos computadores para melhorar a prática e o cuidado a ser prestado ao paciente, minimizando erros, ajudando na elaboração do planejamento da assistência de enfermagem e o acesso rápido e seguro das informações ${ }^{(3)}$.

Outras investigações realizadas no final da década de 80 mostraram o desenvolvimento de diferentes softwares para ajudar as enfermeiras a identificarem os diagnósticos de enfermagem e gerar planos de cuidados individualizados $^{(4-7)}$. Esses estudos apontam que, nessa época, muitas instituições de saúde baseavam os planos de cuidados nos diagnósticos médicos, resultando em planos de cuidados de enfermagem que não caracterizavam a individualidade do cliente, portanto, não refletiam preocupações e especificações dos cuidados de enfermagem.

A presente investigação, tendo como tema central a aplicação do software (PROGQUEM) para o planejamento da assistência de enfermagem em uma Unidade de Queimados, foi alicerçada nas considerações sobre Processo de Enfermagem no Modelo Conceitual de Wanda Aguir Horta, adaptado à Taxonomia I Revisada da NANDA - North American Nursing Diagnosis Association ${ }^{(8)}$, na proposta de intervenções de enfermagem de Linda Juan Carpenito $^{(9)}$ e na Classificação de Resultados mencionada na ICNP ${ }^{(10)}$.

Este estudo teve como objetivo avaliar o desempenho do programa (PROGQUEM) para o planejamento da assistência de enfermagem, buscando verificar se esse software permite a execução de todas as fases do processo de enfermagem, qual o tempo gasto para realizar as etapas com auxílio do computador, em especial se os diagnóstico listados estão corretamente redigidos e se as intervenções de enfermagem são corretamente listadas conforme proposto neste estudo.

\section{METODOLOGIA}

A trajetória metodológica escolhida para o alcance do segundo objetivo desta investigação está estruturada no estudo de caso. A escolha desse tipo de abordagem recai sobre sua aplicabilidade para a prática do processo de enfermagem, considerando que as características desse método oferece a participação do indivíduo no estudo. O estudo foi realizado na Unidade de Queimados do Hospital das Clínicas da Faculdade de Medicina de Ribeirão Preto da Universidade de São Paulo (HCFMRPUSP), localizada na Unidade de Emergência.

A amostra foi constituída de 4 pacientes portadores de queimaduras, internados na Unidade de Queimados. Nos critérios de exclusão de sujeitos da pesquisa ficou definido que, neste momento, não fariam parte pacientes menores de 18 anos de idade. Este projeto de pesquisa foi aprovado pelo Comitê de Ética em Pesquisa do HCFMRP-USP. O Termo de Consentimento PósInformação foi assinado pelos referidos sujeitos do estudo, pertencendo ao arquivo da pesquisadora.

A coleta de dados e o exame físico foi realizado pela própria pesquisadora a partir da estrutura do instrumento norteador para a base de dados do software, com base no modelo adaptado das necessidades humanas básicas ${ }^{(8)}$. Esse instrumento teve a finalidade de guiar $\mathrm{e}$ ordenar a coleta de dados, visando obter a avaliação sistematizada do paciente da amostra. 
Essa coleta de dados (entrevista e exame físico) foi realizada no turno da manhã, para que pudéssemos avaliar os locais queimados e suas características. O tempo desse procedimento foi cronometrado para cada sujeito da pesquisa, compreendendo cada instante que a pesquisadora estava presente, ao lado do paciente. Outras informações foram extraídas do prontuário e confirmadas junto aos sujeitos da pesquisa. Em seguida, dirigimo-nos ao computador para documentar essas informações e processar os dados no software (PROGQUEM), uma vez que tal equipamento ainda não se encontra ao lado do leito do paciente.

O tempo para processar o sistema automatizado (PROGQUEM) também foi cronometrado. Pretendemos com isso mostrar que, com o auxílio do computador, é possível reduzir significativamente o tempo no registro do processo de enfermagem.

O software (PROGQUEM) consiste de 12 telas ativas para executar o planejamento da assistência de enfermagem. Cada tela desenvolvida foi construída para agilizar o processamento dos dados avaliados e identificados na coleta de dados junto ao paciente queimado.

\section{RESULTADO E DISCUSSÕES}

Nossa experiência com o exercício de coleta de dados é de longa data. O domínio da enfermeira sobre a estrutura da coleta de dados, sobre o modelo conceitual adotado, o conhecimento das ciências aplicadas à enfermagem, a especificidade da clientela a ser atendida, entre outras habilidades interpessoais, contribuem para a aplicação do modelo na prática clínica. Considerando o modelo adaptado das necessidades humanas básicas ${ }^{(8)}$, utilizado nesta investigação, percebemos que, em qualquer fase e tempo que se encontra o paciente portador de queimaduras, mesmo após sua alta hospitalar (durante os retornos no ambulatório), essa estrutura proposta para a coleta de dados responde às necessidades de avaliação pela enfermagem.

Quando o profissional é chamado para identificar uma condição de saúde, ele inicia um processo de julgamento, comumente denominado diagnóstico, momento em que fatos e observações são coletados e analisados para determinar associações semelhantes que, por sua vez, são comparadas a conhecimentos prévios e experiências $^{(11)}$.
Para iniciar a coleta de dados houve, com os quatro pacientes, doze encontros, sendo três para cada um. O primeiro encontro foi caracterizado pela apresentação da pesquisadora, com a proposta do estudo e a obtenção do termo de consentimento pós-informação. No segundo encontro foi realizada a entrevista e o exame físico, com duração de: paciente $n^{\circ} 1$ - 32 minutos; paciente $n^{\circ} 2-29$ minutos; paciente $n^{\circ} 3-40$ minutos e finalmente paciente $\mathrm{n}^{\circ} 4 \mathrm{com} 28$ minutos, sendo em média aproximada de 32 minutos para cada paciente. Por ocasião do terceiro encontro, foram realizadas avaliações dos resultados obtidos, após 24 horas.

Após a identificação das necessidades básicas afetadas nos pacientes portadores de queimaduras, procedemos ao registro dessas informações no software (PROGQUEM). A execução desse programa começa com a Tela Inicial, onde selecionamos o ícone Paciente, em seguida exibe-se a tela Selecionar Pacientes, expondo ao usuário os nomes dos pacientes, ou se necessário, poderá incluí-los. O programa PROGQUEM organiza numericamente os pacientes que começam a ser introduzidos no sistema. A abertura dessa tela é rápida, e a interação com o usuário se faz por comandos com o apontador do mouse ou com a seta (para baixo ou para cima - teclado), como foi descrito anteriormente.

O preenchimento da tela Dados pessoais dos pacientes é obrigatória. $\mathrm{O}$ enfermeiro precisa digitar o nome e sobrenome do paciente, número de registro hospitalar (obrigatoriamente), data de nascimento, o endereço e telefones para contatos. As demais informações são completadas com auxílio mecânico do mouse ou da seta (teclado). Notou-se que, durante o preenchimento dos 4 casos estudados, é necessária destreza e noção mínima do usuário com o teclado e/ou mouse. A destreza na digitação facilita muito, interferindo no tempo transcorrido para completar as etapas subseqüentes. Caso ocorra algum erro na informação obtida, é possível restaurar os dados, clicando no botão <Restaurar>. Nos casos estudados não foi preciso utilizar esse botão.

Ao procedermos ao preenchimento e análise da tela relativa aos Dados pessoais do paciente, observamos a ausência de dados referentes à procedência, profissão, estado civil, religião, nome de pai e mãe, que são importantes, principalmente quando se tratar de cadastramento de crianças. $\mathrm{Na}$ escolaridade também poderá ser incluído o nível de informação se é completo ou incompleto. Esses dados 
serão incluídos em nova versão do software, uma vez que esses dados devem ser os mais completos possíveis. Determinadas informações poderão ser coletadas em momentos distintos, no dia-a-dia o programa permite que as informações sejam completadas e modificadas, possibilitando o estabelecimento de novos diagnósticos.

O preenchimento da tela Dados relativos ao acidente foi significativamente fácil. Notou-se que a ordenação de cada campo corresponde à sistemática das informações obtidas junto ao paciente, representando a transcrição da prática clínica. Na tela Dados pessoais do paciente e Dados relativos ao acidente, o sistema permite que o usuário crie uma cópia impressa dessas informações na forma de uma história de enfermagem que pode ser incluída no prontuário do paciente.

Após realizarmos a etapa de coleta de dados, tínhamos em mãos uma grande quantidade de informações. E para interpretar essas informações com exatidão, utilizamos o processo de raciocínio diagnóstico de análise e síntese ${ }^{(11-12)}$. Essa proposta para o processo de raciocínio diagnóstico compreende as seguintes etapas: a) fase de análise - compreende a categorização de dados e a identificação de dados divergentes ou lacunas; b) fase de síntese - identifica-se o processo de raciocínio com o agrupamento dos dados nos padrões adotados, a comparação dos padrões com normas e teorias, modelos da literatura, a inferência ou rolagem de hipótese e finalmente o relacionamento dos fatores causais ou das causas relacionadas à inferência encontrada.

Na fase de análise, a categorização dos dados começa durante a coleta de dados, respeitando-se o modelo escolhido, é um processo que nesse estudo acontece mentalmente. Na fase de síntese, começamos a agrupar os dados mentalmente, realizando uma comparação com o modelo teórico adotado. É exatamente nesse ponto que estabelecemos a categoria de necessidades humanas que estarão afetadas. As etapas que se seguem atendem ao processo do raciocínio diagnóstico na sua fase de síntese, conforme o processo de raciocínio proposto ${ }^{(11-12)}$.

Durante o preenchimento da tela que corresponde Categorias diagnósticas: seleção das necessidades básicas afetadas referentes, foi possível verificar essa relação ao modelo selecionado. Em nosso caso, usando o modelo adaptado das necessidades humanas básicas ${ }^{(8)}$, identificamos nos 4 sujeitos do estudo a categoria de necessidade psicobiológica alterada, assim como a categoria de necessidade psicossocial. Não identificamos nesses sujeitos alterações das necessidades psicoespirituais. O programa PROGQUEM ajuda nessa escolha por ter a descrição, no campo de ajuda, a definição desse grande grupo de necessidades humanas.

Ao analisarmos a execução da tela subcategoria diagnóstica, apreende-se que na categoria de necessidade psicobiológica identificamos 5 subcategorias, sendo: termorregulação, integridade tecidual, sensopercepção, sono e repouso, atividade física e higiene. $\mathrm{Na}$ categoria de necessidade psicossocial, identificamos a subcategoria de segurança e integração social, respeitando o modelo proposto.

A natureza da clientela estudada e suas condições de saúde exercem forte influência na decisão sobre a subcategoria diagnóstica, estando estruturados e bem definidas as comparações nos modelos e conceitos adotados. Observando os pacientes portadores de queimaduras, dependendo do tempo pós-trauma e das suas condições de saúde, ficam em evidência os problemas quando são conhecidos, norteando a próxima etapa a ser identificada pelo enfermeiro.

Diante do raciocínio diagnóstico, fazemos aqui a escolha, ou seja, a inferência ou hipótese diagnóstica. $O$ programa apresenta um campo de ajuda para o usuário identificar a definição do diagnóstico selecionado, tela diagnóstico de enfermagem. Isso ajuda a direcionar a hipótese.

Identificamos 12 diagnósticos de enfermagem distintos, para os quatro pacientes, totalizando 36 diagnósticos (31 de necessidades psicobiológicas e 6 para psicossocial) na amostra estudada. Isso significa média de 9 diagnósticos de enfermagem por paciente. Os 12 diagnósticos de enfermagem identificados na amostra foram: para as necessidades psicobiológicas - risco para temperatura corporal alterada, integridade da pele prejudicada, risco para infecção, dor, distúrbio do padrão do sono e repouso, mobilidade física prejudicada, déficit de auto-cuidado alimentação, déficit de auto-cuidado higiene corporal, déficit de auto-cuidado vestir-se e/ou arrumar-se, déficit de auto-cuidado higiene íntima e para as necessidades psicossociais - ansiedade e interação social prejudicada.

Uma vez feito isso, temos que validar essas inferências, através da identificação no computador, na 
tela Características Definidoras, identificando os sinais e sintomas apresentados pelos pacientes avaliados ou fatores de risco e as causas desses problemas na tela Fatores Relacionados. Assim vamos proceder para cada diagnóstico de enfermagem identificados na amostra estudada. Consideramos importante afirmar que essa etapa é feita individualmente para cada inferência diagnóstica.

O programa PROGQUEM oferece a possibilidade de incluir novas informações referentes a qualquer estrutura apresentada nas telas. Entretanto, não executa a exclusão dos itens já incluídos ou aqueles que forem incluídos. Esse código de fonte pode ser acessado somente pelas pessoas responsáveis pelo programa, com a certeza do que será executado.

O programa exibe posteriormente, para cada lista de diagnóstico, a tela Intervenções de Enfermagem. $O$ PROGQUEM apresenta uma lista das possíveis intervenções de enfermagem ${ }^{(3)}$. Dessas diretrizes de intervenções já elaboradas na literatura, e outras introduzidas pela pesquisadora, no programa, selecionamos para os pacientes da amostra um total de 158 intervenções de enfermagem, com média aproximada de 39 por paciente, considerando todos os diagnósticos identificados. A inclusão de novas intervenções de enfermagem no software, pela pesquisadora, é específica à clientela estudada.

Após a revisão dos diagnósticos de enfermagem identificados e das intervenções de enfermagem selecionadas através da exposição das telas dos relatórios, o software possibilitou imprimir a lista dos diagnósticos de enfermagem e das intervenções de enfermagem. A fase do processo de enfermagem, que inclui a implementação, avaliação e resultados, é executada em texto livre no computador.

Essa aplicação do processo de enfermagem na realidade clínica, em quatro casos reais de pacientes portadores de queimaduras, oferece benefícios para a administração da assistência de enfermagem. Tendo vivenciado todas essas etapas de construção do processo de enfermagem de forma manuscrita durante vários anos, a dificuldade maior que encontrava estava no agrupamento dos dados para redigir corretamente os diagnósticos de enfermagem e as intervenções de enfermagem, de maneira ordenada e que respondesse ao modelo teórico adotado.

Durante a aplicabilidade do software para os casos clínicos apresentados, cronometramos essa implementação. A Tabela 1 mostra o perfil dos diagnósticos identificados nesses sujeitos da pesquisa, o número de intervenções de enfermagem descritas, em comparação com o tempo de execução das etapas no computador.

Tabela 1 - Demonstração do perfil dos diagnósticos de enfermagem identificados no estudo, o número de intervenções de enfermagem descritas, em comparação com o tempo de execução das etapas no computador

\begin{tabular}{cccccc}
\hline $\begin{array}{c}\mathrm{N}^{\circ} \\
\text { pac. }\end{array}$ & $\begin{array}{c}\text { Necessidade } \\
\text { psicobiológica }\end{array}$ & $\begin{array}{c}\text { Necessidade } \\
\text { psicossocial }\end{array}$ & $\begin{array}{c}\text { Necessidade } \\
\text { psicoespiritual }\end{array}$ & $\begin{array}{c}\mathrm{N}^{\mathrm{o}} \text { de interv. } \\
\text { de } \\
\text { enfermagem }\end{array}$ & $\begin{array}{c}\text { Tempo } \\
\text { (minutos) }\end{array}$ \\
\hline 1 & 6 & 1 & 0 & 32 & 16 \\
2 & 6 & 1 & 0 & 26 & 15 \\
3 & 9 & 2 & 0 & 41 & 13 \\
4 & 10 & 1 & 0 & 59 & 14 \\
\hline
\end{tabular}

Alguns estudos mostram os benefícios de um programa automatizado para o planejamento dos cuidados de enfermagem comparando o tempo médio estimado quando realizado de forma manual (manuscrito), sendo de 50 minutos e depois passando a ser realizado por um programa de computador, ficando com um tempo médio de 15 minutos $^{(4)}$.

Nesse estudo observamos que, via de regra, os resultados encontrados foram semelhantes. Nos estudos realizados pela pesquisadora em 1993, consta que o tempo despendido no registro manual da coleta de dados de pacientes queimados, no momento de sua admissão, foi em média de 28,7 minutos ${ }^{(13)}$. Usando-se o computador, notou-se que o tempo para executar e finalizar o programa foi em média de 14,3 minutos. Com o futuro aperfeiçoamento desse programa e do aumento da destreza e habilidade do usuário na utilização do mesmo, esse tempo poderá ser reduzido. Baseada nessas informações, isso representa economia de tempo em registros de enfermagem.

Os diagnósticos de enfermagem computadorizados resultam em documentação da redação de forma correta dos seus enunciados e, estando alicerçados teoricamente, fortalecem a compreensão dos problemas identificados nos pacientes, fornecem uma linguagem científica para o enfermeiro em qualquer uma das atividades dessa profissão, seja na área do ensino, da pesquisa ou da assistência.

A redação manual das afirmativas diagnósticas, realizada na Unidade de Queimados, foi tema de um trabalho de pesquisa desenvolvido em 1996 mostrando 
resultados com erros nos enunciados dos diagnósticas de enfermagem de pacientes portadores de queimaduras, listados pelos enfermeiros. Essas autoras observaram a utilização de expressões inadequadas na união do título com o fator relacionado e desses com as características definidoras $^{(14)}$

Dessa forma, reiteramos que, ao descrever o estado de saúde real do paciente, a afirmativa diagnóstica deve conter 3 partes: o problema (título diagnóstico), a etiologia (fator relacionado) e o sintoma (características definidoras). O formato para descrever os diagnósticos de risco deve contemplar duas partes: o problema (título diagnóstico) e o fator de risco.

Nota-se que descrever os diagnósticos de enfermagem diariamente torna-se complicado. A complexidade que envolve, em especial, essa etapa do processo de enfermagem exige da enfermeira a capacidade de processar rapidamente informações dos pacientes, agrupá-las e estruturá-las de forma que fique claro para toda a equipe de enfermagem os problemas apresentados pelo paciente.

O software aqui apresentado, aplicado numa situação clínica junto ao paciente portador de queimaduras internado, proporcionou a redação das afirmações diagnósticas estruturadas conforme o referencial adotado, portanto, livre de possíveis erros nessa estrutura, discriminou, também, as intervenções de enfermagem para cada diagnóstico identificado.

Uma prescrição de enfermagem organizada de acordo com os diagnósticos de enfermagem identificados, estando alicerçada num referencial teórico, intensifica a qualidade da informação que deve gerar cuidados de enfermagem precisos, muitas vezes complexos, conforme a clientela que está sendo assistida. Acreditamos que a forma melhorada na redação das intervenções de enfermagem possa adequar as habilidades da equipe de enfermagem em providenciar cuidados mais efetivos e consistentes junto ao paciente.

A vantagem tecnológica da automatização dos dados para o planejamento dos cuidados de enfermagem é o acesso que o usuário tem com rapidez e fidelidade das informações.

Uma estrutura metodológica, fundamentada em referenciais teóricos de enfermagem, conciliada com a tecnologia computacional é possível de ser associada. A integração dessa ciência na enfermagem aplica-se ao desenvolvimento de software que resulta em qualidade de trabalho, segurança para o paciente e melhoria dos níveis de formação e competência profissional.
Para utilizar o programa PROGQUEM, é preciso antes de mais nada que o enfermeiro domine as etapas do processo de enfermagem, que conheça os diagnósticos de enfermagem da NANDA ${ }^{(15)}$ e seus componentes. Estar familiarizado com as etapas do processo de enfermagem, associado ao referencial teórico adotado, é hoje uma atividade chave para o enfermeiro promover o pensamento crítico no desempenho da assistência de enfermagem ao seus pacientes. Acreditamos que isso é individual para cada enfermeiro, independente se realiza a documentação do processo de enfermagem com ou sem o auxílio de um computador.

\section{CONSIDERAÇÕES FINAIS}

O sistema foi desenvolvido inicialmente como uma versão $\beta$ para extrair informações do instrumento de coleta de dados de enfermagem, e com isso gerar uma lista de diagnósticos de enfermagem, a prescrição de enfermagem e resolução de enfermagem para cada paciente internado na Unidade de Queimados. Acreditamos que trabalhar com essa clientela altamente definida facilitará os processos de desenvolvimento e refinamento do sistema.

Nos próximos desenvolvimentos, esses dados contidos no sistema permitirão a identificação detalhada da base do conhecimento dos diagnósticos de enfermagem apresentados em cada fase de evolução do paciente queimado. À medida que o sistema entra em uso, novos dados coletados serão adicionados, refinando e expandindo a base do conhecimento para a prática de enfermagem.

Inúmeras aplicações na pesquisa do Processo de Enfermagem são possíveis usando o sistema automatizado PROGQUEM. Embora muitas metodologias possam ser usadas para se fazer pesquisa sobre processo de enfermagem sem computadores, um sistema como o PROGQUEM pode capacitar os pesquisadores a obterem tamanhos maiores de amostras, dados e informações com investimento mínimo de tempo e maior fidelidade dos dados, caracterizando o aperfeiçoamento da qualidade do cuidado a ser prestado.

Para implementar sistemas informatizados é importante estudar as operações do dia-a-dia, ou seja, repensar as atividades diárias como regra básica, podendo, assim, compreender todo o processo de trabalho da enfermagem, para então instituir estratégias que viabilizem a prestação do cuidado ${ }^{(16)}$. 


\section{REFERÊNCIAS BIBLIOGRÁFICAS}

1. Evora YDM. Processo de informatização em enfermagem: orientações básicas. São Paulo: EPU; 1995.

2. Conselho Federal de Enfermagem (BR). Documentos Básicos de Enfermagem. Brasília: COFEn; 1997.

3. Evora YDM, Scochi CGS, Nakao JRS, Rodrigues RAP, Fávero N. O computador nas unidades de internação de um hospital escola - expectativa do enfermeiro. Rev Hosp Admin Saúde 1990 abr/jun; 14(2):83-7.

4. Bailey DR. Computer Applications in Nursing: a prototypical model for planning nursing care. Comput Nurs 1988 Sept/ Oct; 6(5):199-203.

5. Bloom KC, Leitner JE, Solano JL. Development of na Expert System Prototype to Generate Nursing Care Plans Based on Nursing Diagnosis. Comput Nurs 1987 Jul/Aug; 5(4):140-5. 6. Chang BL, Roth K, Gonzales E, Caswell D, DiStefano J. A knowledge, based system nursing diagnosis. Comput Nurs 1988 Jan/Fev; 6(1):13-21.

7. Hinson DK, Bush C. Corporate standards for nursing care: an integral part of a computerized care plan. Comput Nurs 1988 Jul/Aug; 6(4):141-6.

8. Rossi LA, Dalri MCB. Processo de enfermagem numa unidade de queimados: análise e proposta de reformulação segundo o modelo conceitual de Horta e taxonomia I dos diagnósticos de enfermagem da NANDA. Rev Esc Enfermagem USP 1993; 27(3):328-54.

9. Carpenito LJ. Diagnósticos de enfermagem: aplicação à prática clínica. Porto Alegre (RS): Artes Médicas; 1997.

10. International Council of Nurse. Introducing ICN's international classification for nursing practice (ICNP): a unifying framework. Int Nurs Rev 1996; 43(6):169-70.

11. Carvalho EC de, Jesus CA de. Raciocínio clínico na formulação de diagnósticos de enfermagem para o indivíduo. In: Antunes MJM, Silva MTN. O uso do diagnóstico na prática da enfermagem. Brasília: ABEn; 1997. p.27-38.

12. Risner PB. Diagnosis: analysis and synthesis of data. In: Christensen PJ, Kenney JW. Nursing process: application of conceptual models. 2nd St. Louis: CV Mosby; 1986. p.124-50. 13. Dalri MCB. Perfil diagnóstico de pacientes queimados segundo o modelo conceitual de Horta e Taxonomia I Revisada da NANDA. [dissertação]. Ribeirão Preto (SP): Escola de Enfermagem de Ribeirão Preto/USP; 1993.

14. Dalri MCB, Rossi LA, Garcia TR, Carvalho EC. Diagnóstico de enfermagem em uma unidade de queimados: análise estrutural dos enunciados. Rev Bras Enfermagem 1996 jan/ mar; 49(1):7-16.

15.North American Nursing Diagnosis Association. Nursing Diagnosis: definitions \& classification 1997-1998. Philadelphia; 1996.

16. Évora, YDM. O computador a beira do leito. Rev Latino-am Enfermagem 1999 dezembro; 7(5):133-4. 